\title{
It Ain't Necessarily So: Interpretations and Misinterpretations of Quantum Theory
}

\author{
John Stachel* \\ Center for Einstein Studies, Boston University \\ E-mail: john.stachelegmail.com
}

The traditional view is that a theory is a conceptual framework providing predictions, and the results of experiments or observations decide whether the theory is right or wrong. It will be contrasted with the modern view that one must incorporate the conditions of applicability of a concept into the very meaning of the concept (measurability analysis), and that only a series of theories (scientific research program) can be said to be scientific or unscientific. This modern view will be applied to a number of questions in quantum mechanics (what is quantization?, states vs processes, open vs closed systems) and quantum field theory (particles and field quanta, bosons vs fermions), and to the search for a theory of quantum gravity (background independent vs fixed background theories).

Frontiers of Fundamental Physics 14 - FFP14,

15-18 July 2014

Aix Marseille University (AMU) Saint-Charles Campus, Marseille

\footnotetext{
*Speaker.
} 